\title{
Fiebre mediterránea familiar. Nueva mutación MEFV con presentación clínica inusual
}

\author{
Francisco Romero-Santana', Loida García-Cruz², Nieves Jaén-Sánchez', Miguel Hervás-García³, Alfredo Santana-Rodríguez², José \\ Luis Pérez-Arellano' \\ 'Unidad de Enfermedades Infecciosas y Medicina Tropical. Servicio de Medicina Interna. Complejo Hospitalario Universitario Insular Materno \\ Infantil (CHUIMI). Las Palmas de Gran Canaria (Las Palmas). España \\ 2Unidad de Genética Clínica. Complejo Hospitalario Universitario Insular Materno Infantil (CHUIMI). Las Palmas de Gran Canaria (Las Palmas). \\ España
}

${ }^{3}$ Servicio de Neurología. Complejo Hospitalario Universitario Insular Materno Infantil (CHUIMI). Las Palmas de Gran Canaria (Las Palmas). España

Recibido: 08/10/2018

Aceptado: 14/11/2018

En línea: 31/12/2018

Citar como: Romero-Santana F, García-Cruz L, Jaén-Sánchez N, Hervás-García M, Santana-Rodríguez A, Pérez-Arellano JL. Fiebre mediterránea familiar. Nueva mutación MEFV con presentación clínica inusual Rev Esp Casos Clin Med Intern (RECCMI). 2018 (Dic); 3(3): 127-129. doi: 10.32818/reccmi.a3n3a9.

Autor para correspondencia: Francisco Romero-Santana. franciscojromerosantana@gmail.com

\section{Palabras clave}

$\triangleright$ Fiebre mediterránea familiar

$\triangleright$ Mielitis transversa

$\triangleright$ Canarias

\section{Resumen}

La fiebre mediterránea familiar es una enfermedad autoinflamatoria caracterizada por episodios recurrentes de fiebre e inflamación estéril de membranas serosas, articulaciones y/o piel. El gen afectado es MEFV, que codifica una proteína denominada pirina o "marenostrina". En este trabajo comunicamos una familia cuyo caso índice fue el de una mujer joven con una mielitis transversa como la primera manifestación verificada genéticamente, presentando una mutación en el gen MEFV [p.Pro180Arg] no descrita previamente y con un patrón de herencia autosómico dominante, asociada a una mutación heterocigota del factor $\vee$ Leiden.

Abstract
Familial Mediterranean fever is an autoinflammatory disorder characterized by recurrent bouts of fever and sterile
inflammation mostly confined to serosal membranes, joints, and/or skin. The defective gen is MEFV that encodes a
protein known as pyrin or also "marenostrin". Here, we describe a case of a young woman with a transverse myelitis
as the first manifestation of genetically verified disease, presenting a novel mutation in the MEFV gene and an
autosomal dominant pattern of inheritance, associated with an heterozygous Leiden factor V mutation.

\section{Puntos destacados}

$\triangleright$ Se trata del primer paciente comunicado con fiebre mediterránea familiar en las Islas Canarias.

$\triangleright$ Los hallazgos neurológicos han sido raramente descritos en pacientes con fiebre mediterránea familiar.

$\triangleright$ Patrón de transmisión autosómico dominante del gen MEFV en esta familia.

$\triangleright$ Posible relación entre las alteraciones de la coagulación y las manifestaciones clínicas.

\section{Introducción}

La fiebre mediterránea familiar (FMF) es la forma de fiebre periódica hereditaria más prevalente. Su prevalencia es variable y depende de factores étnicos (es mayor en judíos sefarditas y turcos) $)^{1}$. Generalmente, se describe como una enfermedad autosómica recesiva que se caracteriza por episodios cortos y recurrentes de fiebre y dolor secundario a serositis (peritoneo, pleura, peri- cardio, sinovial y túnica vaginal testicular). La piel y el tejido muscular también pueden estar afectados con lesiones erisipeloides o nodulares, y mialgias con menor frecuencia ${ }^{2}$. El diagnóstico de confirmación se realiza, al menos, con la detección de variantes patogénicas en el gen MEFV, localizado en el cromosoma 16p13.3. Este gen codifica para una proteína conocida como marenostrina/pirina que inhibe la activación de la caspasa 1, disminuyendo el proceso de inflamación. La alteración de esta proteína en los neutrófilos se asocia a un aumento de la interleucina $1 \beta$, produciendo una activación descontrolada de estas células y promoviendo su migración a las membranas serosas ${ }^{3-5}$. Las manifestaciones neurológicas en la FMF raramente han sido publicadas, y su presentación es muy heterogénea (convulsiones, trombosis de senos venosos, pseudotumor cerebri, neuritis óptica, complicaciones del sistema nervioso central asociadas a vasculitis sistémicas, lesiones desmielinizantes y esclerosis múltiple (EM), ictus isquémico y meningitis aséptica recurrente) ${ }^{6,}$ ? . El empleo de colchicina constituye el primer escalón terapéutico en la FMF, reservando los moduladores de respuesta biológica (anakinra, canakinumab y rilonacept) para situaciones con respuesta insuficiente o intolerancia a la colchicina ${ }^{1,4}$. 
Romero-Santana F, García-Cruz L, Jaén-Sánchez N, Hervás-García M, Santana-Rodríguez A, Pérez-Arellano JL.

Fiebre mediterránea familiar. Nueva mutación MEFV con presentación clínica inusual

En esta publicación, describimos el caso índice de una mujer joven con una mielitis transversa como la primera manifestación de una FMF verificada genéticamente, presentando una mutación en el gen MEFV no descrita previamente y con un patrón de herencia autosómico dominante, asociada a una mutación heterocigota del factor $V$ Leiden y a una deficiencia de proteína S.

\section{Historia clínica: antecedentes, enfermedad actual y exploración física}

Se trata de una mujer caucásica de 19 años sin consumo previo de fármacos o tóxicos y con historia de dermatitis atópica, distonía mandibular secundaria a metoclopramida e infertilidad. En relación con este último problema, se realizó un estudio de hipercoagulabilidad en el que se constató una mutación heterocigota para el factor $\vee$ Leiden y una deficiencia de proteína S. Inicialmente, fue derivada al Servicio de Urgencias por dolor abdominal, fiebre, mialgias y cefalea de 2 semanas de duración, acompañado de dolor, hipoestesia, parestesias y debilidad muscular en las extremidades inferiores. Posteriormente, la paciente desarrolló retención aguda de orina secundaria a disfunción vesical. No se observaron hallazgos patológicos en la resonancia magnética cerebral y de columna cervical, dorsal y lumbar, descartándose lesión compresiva. El líquido cefalorraquídeo era claro, sin células, con glucorraquia normal y discreta proteinorraquia. Se realizó un estudio electrofisiológico que, junto con los datos previos, permitió realizar el diagnóstico de mielitis transversa con radiculitis izquierda L5-S1. La paciente fue tratada con bolos de $500 \mathrm{mg}$ de metilprednisolona, con respuesta parcial.

Pocos meses después de ser dada de alta del Servicio de Neurología, consultó por fiebre, presencia de placas eritematoedematosas con morfología anular en el tronco (Figura 1) y elevación de los reactantes de fase aguda en la analítica (leucocitos 21.300/ $\mu$ l con 92\% de neutrófilos). La paciente refería episodios de un año de duración, aproximadamente cada 4 semanas, caracterizados por cefalea y astenia, y acompañados de fiebre de $38-39^{\circ} \mathrm{C}$. Estos episodios eran desencadenados por el estrés y asociados a dolor abdominal, vómitos y diarrea. No se objetivó líquido libre intraabdominal en el momento agudo. Esta misma sintomatología estaba presente en su madre, su abuelo materno y su tía materna.

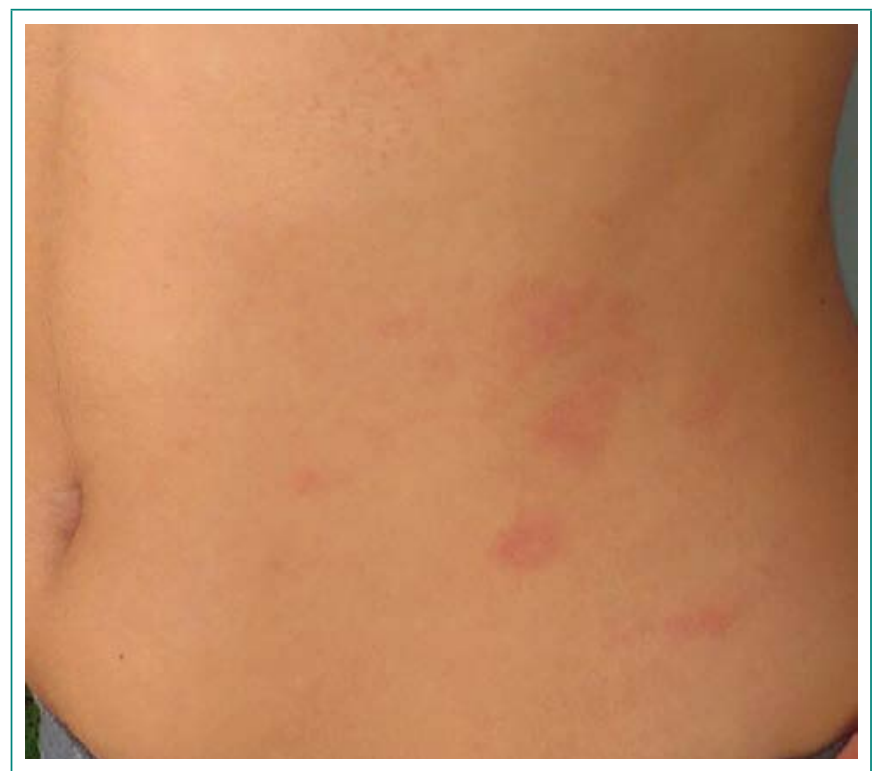

Figura 1. Lesiones cutáneas en el abdomen

\section{Evolución}

Con la sospecha clínica de FMF, se procedió a realizar un estudio genético completo. Se realizó secuenciación tradicional (Sanger) de toda la zona codificante y regiones intrónicas flanqueantes de los genes TNFRS1A y MEFV. Sólo fue detectada una variante anormal en el gen MEFV. El estudio genético familiar (Figura 2) reveló que la misma mutación se encontraba presente, en heterocigosis, en su madre (II:1), su abuelo materno (l:1) y su tía materna (II:3)

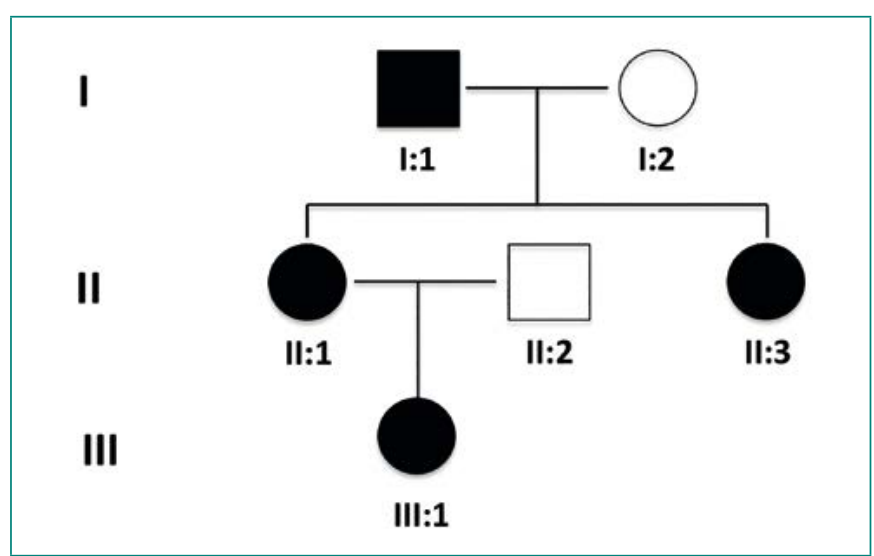

Figura 2. Árbol genealógico del caso índice (III:1) que incluye a su madre (II:1), abuelo materno (I:1) y tía materna (II:3), todos ellos con manifestaciones clínicas

Tras el diagnóstico genético, se inició tratamiento con colchicina (1g/día), con mejoría de los episodios inflamatorios. Siguiendo el protocolo habitual, el consejo genético pre y post-estudio fue realizado en la consulta de la Unidad de Genética Clínica durante el cual se recopilaron los consentimientos informados necesarios.

\section{Diagnóstico}

Fiebre mediterránea familiar con patrón de herencia autosómico dominante. Mielitis transversa como primera manifestación de la enfermedad con mutación heterocigota del factor $\mathrm{V}$ Leiden y deficiencia de proteína S asociados.

\section{Discusión y conclusiones}

Presentamos el caso de una paciente joven con mielitis transversa e historia de fiebre periódica a la que se realizó un despistaje mutacional mediante la secuenciación de los genes MEFV y TNRFS1A. Este estudio descubrió la variante heterocigota c.539C>G (p.(Pro180Arg)) en el exón 2 del gen MEFV. Se trata de una nueva mutación que ha sido registrada, pero no publicada con anterioridad en asociación con la FMF. Aunque no existen ensayos funcionales que aclaren con certeza su potencial patogenicidad, los diferentes predictores bioinformáticos usados la designan como tal. Asimismo, la variante no está presente en, al menos, 300 pacientes sanos. Este cambio de nucleótido sustituye el aminoácido prolina por arginina en la posición 180 de la proteína, alterando la función normal de la pirina.

La mielitis transversa puede ser debida a varias causas, aunque lo más frecuente es que aparezca como un fenómeno autoinmune tras una infección o vacunación, como resultado de una infección directa, un proceso autoinmune sistémico subyacente, o una enfermedad desmielinizante adquirida ${ }^{8}$. 
Algunos estudios apoyan la asociación entre la FMF y enfermedades desmielinizantes, especialmente la EM, algo esperable, ya que ambas entidades comparten episodios agudos de inflamación. En el estudio de Akman Demir y col., la prevalencia de FMF en una cohorte turca de EM fue cuatro veces superior a la esperada en la población general de Turquía?. Por otro lado, otros autores encontraron un aumento significativo en la prevalencia de portadores de mutaciones en MEFV entre los pacientes con EM, comparado con sujetos sanos (38\% y $11 \%$, respectivamente) 7 . Estos datos sugieren que las alteraciones en la secuencia de MEFV predisponen al desarrollo de una enfermedad desmielinizante. El mecanismo de este efecto es desconocido, aunque la correlación entre la actividad de la enfermedad en la EM y la liberación de la caspasa-1 dependiente de interleucina $1 \beta$ por parte de las células mononucleares en la sangre periférica de estos pacientes sugeriría una vía común ${ }^{6}$. En este caso, la paciente no cumplía criterios de EM y las analíticas para autoinmunidad fueron todas negativas.

Por otro lado, en esta familia, el patrón de transmisión corresponde a una forma autosómica dominante, a pesar de que la FMF se describe tradicionalmente como una enfermedad con un patrón autosómico recesivo. Este patrón de herencia está siendo cuestionado por algunos estudios en los que, hasta el 40\% de los pacientes diagnosticados clínicamente de FMF en los países occidentales son portadores de un solo alelo mutado. Booth y col. estudiaron a cinco familias con la mutación en el gen MEFV y un patrón de herencia autosómico dominante. En tres de ellas, tras descartar otros síndromes de fiebre periódica, se encontró un verdadero patrón de herencia autosómico dominante con una penetrancia variable ${ }^{10}$. Todo ello sugiere que la FMF tiene una base genética heterogénea y aún no bien definida².

Aunque la FMF afecta predominantemente a personas de la cuenca del Mediterráneo', no existen datos acerca de su epidemiología en España debido a su baja prevalencia4. En España, ha sido descrita en un grupo de personas (82 casos) entre los descendientes de judíos conversos en Mallorca y casos aislados en Gerona, Toledo, Madrid y Andalucía ${ }^{2}$. Tras una amplia revisión bibliográica, consideramos que es el primer caso descrito en las Islas Canarias.

Por último, el aumento del ambiente inflamatorio en la FMF puede predisponer a la alteración en las pruebas de coagulación como resultado de la inflamación crónica subclínica7.11. Sin embargo, aunque se ha observado una tendencia a la hipercoagulabilidad en diferentes cohortes de pacientes con FMF, sólo se han publicado casos anecdóticos de manifestaciones trombóticas en ausencia de amiloidosis o síndrome nefrótico ${ }^{6}$. La paciente presentaba una mutación heterocigota del factor $V$ Leiden, un hallazgo no publicado anteriormente en asociación a la FMF y que podría explicar su infertilidad. Por otro lado, en la FMF se puede producir infertilidad debido a las adherencias abdominopélvicas por peritonitis de repetición y por lesiones ováricas secundarias a depósito amiloide ${ }^{12}$.

\section{Bibliografía}

1. Alghamdi M. Familial Mediterranean fever, review of the literature. Clin Rheumatol. 2017 Aug; 36(8): 1707-1713. doi: 10.1007/s10067-017-3715-5.

2. Buades Reinés J, Aguirre Errasti C. Familial Mediterranean fever. Med Clin (Barc). 2001; 117: 142-146.

3. Ben-Chetrit E, Levy M. Familial Mediterranean fever. Lancet. 1998; 351:659-664.

4. Aróstegui J. Hereditary systemic autoinflammatory diseases. Reumatol Clin. 2011 Jan-Feb; 7(1): 45-50. doi: 10.1016/j.reuma.2010.01.010.

5. Estébanez Muñoz M, Gómez Cerezo J, López Rodríguez M, Pagán Muñoz B, Barbado Hernández FJ. Periodic familial fever. An Med Intern. 2008; 25: 93-97.

6. Rigante D, Lopalco G, Tarantino G, Compagnone A, Fastiggi M, Cantarini L. Non-canonical manifestations of familial Mediterranean fever: a changing paradigm. Clin. Rheumatol. 2015 Sep; 34(9): 1503-1511. doi: 10.1007/ s10067-015-2916-z

7. Feld O, Yahalom G, Livneh A. Neurologic and other systemic manifestations in FMF: published and own experience. Best Pract Res Clin Rheumatol. 2012 Feb; 26(1): 119-133. doi: 10.1016/j.berh.2012.01.004.

8. Frohman EM, Wingerchuk DM. Transverse myelitis. N Engl J Med. 2010 Aug 5; 363(6): 564-572. doi: 10.1056/NEJMcp1001112.

9. Akman-Demir G, Gul A, Gurol E, Ozdogan H, Bahar S, Oge AE, et al. Inflammatory/demyelinating central nervous system involvement in familial Mediterranean fever (FMF): coincidence or association? J Neurol. 2006; 253: 928-934.

10. Booth DR, Gillmore JD, Lachmann HJ, Booth SE, Bybee A, Soytürk M, et al. The genetic basis of autosomal dominant familial Mediterranean fever. QJM. 2000; 93: 217-221.

11. Demirel A, Celkan T, Kasapcopur O, Bilgen H, Ozkan A, Apak H, et al. Is familial Mediterranean fever a thrombotic disease or not ? Eur J Pediatr. 2008. 167: 279-285

12. Yanmaz MN, Özcan AJ, Savan K. The impact of familial Mediterranean fever on reproductive system. Clin. Rheumatol. 2014; 33(10): 1385-1388. doi: 10.1007/s10067-014-2709-9. 